\title{
Changes in Energy Cost and Total External Work of Muscles in Elite Race Walkers Walking at Different Speeds
}

\author{
by \\ Wiesław Chwała1, Andrzej Klimek², Wacław Mirek
}

The aim of the study was to assess energy cost and total external work (total energy) depending on the speed of race walking. Another objective was to determine the contribution of external work to total energy cost of walking at technical, threshold and racing speed in elite competitive race walkers.

The study involved 12 competitive race walkers aged $24.9 \quad 4.10$ years with 6 to 20 years of experience, who achieved a national or international sports level. Their aerobic endurance was determined by means of a direct method involving an incremental exercise test on the treadmill.

The participants performed three tests walking each time with one of the three speeds according to the same protocol: an 8-minute walk with at steady speed was followed by a recovery phase until the oxygen debt was repaid. To measure exercise energy cost, an indirect method based on the volume of oxygen uptake was employed. The gait of the participants was recorded using the $3 D$ Vicon opto-electronic motion capture system.

Values of changes in potential energy and total kinetic energy in a gate cycle were determined based on vertical displacements of the centre of mass. Changes in mechanical energy amounted to the value of total external work of muscles needed to accelerate and lift the centre of mass during a normalised gait cycle.

The values of average energy cost and of total external work standardised to body mass and distance covered calculated for technical speed, threshold and racing speeds turned out to be statistically significant ( $p$ 0.001).

The total energy cost ranged from $51.2 \mathrm{~kJ} . \mathrm{m}-1$ during walking at technical speed to $78.3 \mathrm{~kJ} . \mathrm{m}-1$ during walking at a racing speed. Regardless of the type of speed, the total external work of muscles accounted for around $25 \%$ of total energy cost in race walking. Total external work mainly increased because of changes in the resultant kinetic energy of the centre of mass movement.

Key words: race walking, energy cost, total energy, maximal oxygen uptake, anaerobic threshold.

\section{Introduction}

Substantial aerobic endurance is one of the key factors underlying athletes' success in endurance sports. In these disciplines, special importance is attributed to maximal oxygen uptake, but particularly to the speed of movement (of running or walking) with exercise intensity at the anaerobic threshold. There are cases, however, that while the variables do not significantly differentiate athletes, their performance is markedly different. This may be due to different energy cost of movement characterising athletes within the same discipline. The value of the variable depends on many factors, mainly on the technique of movement (Klimek and Chwała, 2007).

In the available studies dealing with race walking, energy cost (Klimek and Chwała, 2007) and biomechanical cost (Chwała, 2013) are rarely

\footnotetext{
1 - Institute of Biomedical Sciences, Department of Biomechanics of University School of Physical Education in Cracow, Poland.

2 - Institute of Biomedical Sciences, Department of Physiology and Biochemistry of University School of Physical Education in Cracow, Poland.

3 - Institute of Sport Sciences, Department of Athletics of University School of Physical Education in Cracow, Poland.
} 
analysed. Analysis of the literature shows that the problem of estimating the energy cost of athletes was based mostly on the method of heart rate monitoring (Motonaga et al., 2006). Combining the knowledge accumulated by physiology and biomechanics with the available state-of-the art, computerised measurement devices are hard to overestimate. One of them is the possibility of determining the optimal technique of movement that allows energy cost to be kept at a minimum level.

An increase in walking speed sports, on the one hand, results in myocytes increased demand for oxygen to reach the maximum oxygen uptake, on the other hand raises the share of anaerobic processes in the total energy cost.

A reduction in the amount of energy consumed by working muscles directly diminishes their demand for oxygen, thus decreasing the proportion of anaerobic metabolism without affecting the intensity of exercise. This means that the athlete can move faster consuming the same volume of oxygen.

Race walking is an endurance sports event, with a predominance of aerobic metabolism. In the training process of race walkers, different training modes are used for the improvement of technique (technical speed), aerobic capacity (threshold speed), and for competition (racing speed). The assumptions presented above directly apply to the performance of race walkers. The main element of biomechanical cost is energy used to control the displacements of the centre of mass. If its value is low, then the level of total biomechanical cost is also low.

The main component of the biomechanical cost is energy consumed to control the change of the center of mass of the body. Its low value determines the low total biomechanical cost. According to some authors there is a relationship between the physiological and biomechanical cost of the gait (Ortega and Farley, 2005; Umberger and Martin, 2007), therefore Martina et al. (1992) indicate the absence of such a relationship.

Indirect physiological methods mainly calculate the cumulative metabolic cost, whereas the method of calculating the external work performed by the muscles, characterizes the individual technique of control the center of gravity in walking. It is related to the strategy of the movement, aimed at minimizing cost and maximizing energy recovery (Chwała, 2013).

The purpose of the study was to evaluate the energy cost and the total external work (total energy), depending on the speed of race walking. Another objective was to determine the contribution of external work to total energy cost of walking at a technical, threshold and racing speed in elite competitive race walkers.

\section{Material and Methods}

The study was performed on 12 competitive race walkers representing champion and international champion levels. Their age was 24.9

4.10 years, body height $1.80 \quad 0.68 \mathrm{~m}$, and body mass $697.06 \mathrm{~kg}$. The mean value of the BMI in the group was $21.26 \quad 1.81 \mathrm{~kg} . \mathrm{m}-2$. The training experience of the participants ranged from 6 to 20 years. Some of them were (1-8) Olympic and World Championships finalists and one was a European Championship medallist. The study protocol was approved by the Local Ethical Committee and was performed in accordance with the Declaration of Helsinki.

The experiment protocol required the athletes to perform typical pre-season training of high volume involving aerobic and mixed exercise (140-180 km of race walking per week). Research was carried out in the morning, in an air-conditioned laboratory at 20-21 C. The inclusion criteria for athletes, were to be at least of a champion class race walker, provide current medical examination and individual consent to participate in the study.

The aerobic capacity of the participants (maximal oxygen uptake and of the anaerobic threshold) was determined using a direct method involving an incremental exercise test on a treadmill. The first three minutes of the test were a warm up during which the participants walked at $8 \mathrm{~km} . \mathrm{h}-1$. Then the walking speed was increased by $1 \mathrm{~km} . \mathrm{h}-1$ every three minutes. After consultations with the participants $15 \mathrm{~km} . \mathrm{h}-1$ was accepted as the critical speed, after which the treadmill incline was raised $1 \%$ to increase exercise intensity. The purpose of this regulation was to prevent participants turning swing phase, that is, from racing to run. The test was continued to volitional exhaustion. The level of the anaerobic threshold was calculated from the dynamics of 
changes in the respiratory system parameters (the maximum value of $\mathrm{FECO}$, a significant increase in VE and the minimum value of VE.VCO2-1) (Reinhard et al., 1979).

The purpose of the main component of the experiment was to determine the energy cost of the participants walking with the technical speed vt (when the movement technique can be controlled best), threshold speed - vp (at the anaerobic threshold), and racing speed - vs (used during $20 \mathrm{~km}$ race walk events). The threshold and maximum speeds were determined individually for each athlete during the incremental exercise test; the racing speed was established based on the athletes' performance in $20 \mathrm{~km}$ race walk events, depending on their sport level it ranged from 10 to $12 \mathrm{~km} . \mathrm{h}-1$. In the exercise test constituting the core of the experiment participants walked with three speeds according to the same protocol: three minutes of rest were followed by 8 minutes of walk with a steady speed (vt, vp, vs,) and by another period of rest until oxygen uptake returned to rest values (the oxygen debt was repaid).

During the exercise test, the respiratory and circulatory systems' variables (lung minute ventilation - VE, the percentage content of oxygen - FEO2 and carbon dioxide - FECO2 in the exhaled air, minute oxygen uptake - $\mathrm{VO} 2$ and the volume of carbon dioxide expelled - VCO2, the breathing quotient - $R Q$, the breathing equivalent for oxygen - VE.VO2-1 and carbon dioxide VE.VCO2-1 and heart rate) were recorded for all speeds every 30 seconds.

Exercise energy cost (EE - energy expenditure) was determined by an indirect method based on net oxygen uptake during and immediately after exercise, until all oxygen debt was repaid. The caloric equivalent was used to this end, the value of which was selected in relation to the current ratio between the volume of exhaled carbon dioxide and the volume of oxygen uptake (RER).

The treadmill gait was recorded using a Vicon 250 opto-electronic motion capture system. To enable the acquisition of the measurement data, passive markers were attached to participants' skin in anatomical points as indicated in the producer's manual, in conformity with the selected biomechanics model Golem (by Oxford Metrics Ltd). The markers indicated the dimensions and spatial orientation of body segments and the position of joint centres. The measurement data were recorded with a frame rate of $120 \mathrm{fps}$.

Vertical displacements of the centre of mass $(\mathrm{CoM})$ during a gait cycle were determined using a kinematic method (Eames et al., 1999).

The displacements were used to calculate average values of potential energy changes in relation to the lowest position of the CoM in the gait cycle based on the following formula:

$$
\Delta E_{p}=m_{c} g\left(h_{\max }-h_{\min }\right)[\mathrm{J}],
$$

where: $\Delta \mathrm{Ep}$ - the average value of changes in potential energy of CoM in the cycle [J]; hmax, hmin - respectively the highest and lowest position of CoM in the cycle [m], mc - body mass [kg].

The resultant CoM velocity was calculated in relation to the axes of the spatial system of coordinates using the relationship:

$$
v_{w}=\sqrt{v_{x}^{2}+v_{y}^{2}+v_{z}^{2}} \text { [ms-1], }
$$

where: vx, vy, vz - CoM velocities with respect to particular axes of the spatial system of coordinates.

The resultant CoM velocity average was used to calculate changes in total kinetic energy $\Delta \mathrm{Ek}$ during the gait cycle:

$$
\Delta E_{k}=\frac{m \Delta v_{w}{ }^{2}}{2} \text { [J], }
$$

where: $\Delta \mathrm{EK}$ - average value of changes in the resultant kinetic energy of the body during the cycle [J]; mc - body mass of the particular participant $[\mathrm{kg}] ; \Delta \mathrm{vw}$ - change in CoM velocity.

The value of changes in mechanical energy equivalent to the value of total external energy needed to accelerate and lift the centre of mass in the cycle ("total energy") (Cavagna et al., 2002; Minetti et al., 1994; Schepens et al., 2004) was calculated as a sum of changes in potential energy and resultant kinetic energy of CoM vertical and translational movement according to the following equation:

$$
\Delta E_{c}=\Delta E_{p}+\Delta E_{k} \quad[\mathrm{~J}],
$$

where: $\triangle \mathrm{Ec}$ - average value of changes in total energy of CoM.

In the next step, the average changes in potential, kinetic and total energy were standardised by a kilogram of body mass and one meter of distance covered. 
To determine the body mass and height of the participants the digital Body Composition Analyzer scale TBF-300 manufactured by the Japanese company Tanita and an anthropometer enabling measurements with accuracy of $0.01 \mathrm{~m}$ were respectively used. All exercise test was performed on the Swedish-made treadmill "Cardionics" model 2113. The respiratory system parameters were recorded by a portable ergospirometer Start-2000-M made by MES, which was programmed to take continuous measurements averaged every $30 \mathrm{~s}$. The participants' heart rate was registered by a recording device connected to the ergospirometer at $15 \mathrm{~s}$ intervals.

To capture statistically significant differences between energy cost (EE) and total external work of muscles $(\Delta \mathrm{Ec})$ for successive walking speeds, the repeated measurement ANOVA and the Tukey's post-hoc test were applied. The differences between the average values of energy cost $(\mathrm{EE})$ and total external work $(\triangle \mathrm{Ec})$ were tested for significance by one-way ANOVA, taking the actual walking speed as the dependent variable. To select statistically significantly different pairs of variables in both analyses the Tukey's post-hoc test was applied.

\section{Results}

In the incremental exercise test, the participants reached maximum oxygen uptake of $67.4 \quad 7.25$ mL.kg-1.min-1 (4.7 0.81 L.min-1). This result is characteristic of athletes in endurance sports, who can successfully compete in international events (Table 1). The average lung ventilation at which $\mathrm{VO} 2 \mathrm{max}$ was reached was 139.7 18.8 L.min-1 and the heart rate was 186 10.7 beats per min-1. Walking speed at the termination of the exercise test (at $\mathrm{VO} 2 \mathrm{max}$ ) was 15 km.h-1 (4.2 m.s-1, 250 m.min-1), most frequently at a treadmill incline of $1 \%$.

The average energy cost of walking (EE) per 1 minute of exercise for vt was lower by 27.1 kJ.min-1 than in case of vs. Slightly larger differences of EE were established between the technical and threshold speeds (a difference of $35 \%$ ) than between the threshold and racing speeds (13\%). The average values of EE cost calculated for particular walking speeds were significantly different at $\mathrm{p} \quad 0.001$.

An increase was also found in the biomechanical cost of walking calculated as total external work by 0.89 kJ.min-1 for extreme walking speeds. As in case of physiological cost, the cost increased more between the technical speed and threshold speeds (a difference of 16\%) than between the threshold and racing speeds $(4 \%)$. The differences between the average values of total external work at particular walking speeds (kJ.min-1) were statistically significant at p 0.001, likewise the differences between the physiological cost of EE and total external work expressed in $\mathrm{kJ} . \mathrm{m}-1$ for both the threshold (p 0.05) and racing speed ( $p$ 0.005). However, the average values of energy cost obtained with both methods for the technical speed were not significantly different.

The data in Table 2 show that physiological cost standardised by meter of distance covered increased by 59 J.m-1 for extreme walking speeds. This means that the values of variables increased between successive speeds by, respectively, $17 \%$ and $4 \%$. EE values calculated for vt and vs, and vt and vp were significantly different (at p 0.005 and $\mathrm{p} \mathrm{0.01,}$ respectively). There were no significant differences between the threshold speed and the racing speed.

Total external work per one meter of distance covered increased by $20,4 \mathrm{~J} . \mathrm{m}-1$, so it increased between successive walking speeds by $18 \%$ and $10 \%$, respectively. The average values of total external work per distance covered were significantly different for all walking speeds at $\mathrm{p}$ 0.001. Similar statistical significance of the differences ( $p$ 0.001) was obtained when the average values of $\mathrm{EE}$ and $\Delta \mathrm{Ec}$ standardized by a meter of distance covered were compared.

The values obtained when energy costs additionally were standardised by one kilogram of body mass were compared and an increase by 0,88 J.m-1.kg-1 was observed. The amounts by which average values increased between successive walking speeds were $17 \%$ and $5 \%$, respectively. The increases were significantly different for vt and $v p$ and $v t$ and vs at $\mathrm{p} 0.005$. However, no significant differences between the threshold and racing speeds were noted.

The value of total external work standardised by body mass was higher by $0,3 \mathrm{~J} \cdot \mathrm{m}-1 . \mathrm{kg}-1$ for vs in relation to vt. In relative terms, it increased between the technical speed and the threshold speed by $18 \%$. The difference between the threshold speed and the racing speed was smaller: 
$10 \%$ of the initial value. As in case of total external work standardised by one meter of distance covered, additional standardisation by body mass also produced significant differences for all walking speeds ( $p$ 0.001).
An analysis of differences between the average values of $\mathrm{EE}$ and $\Delta \mathrm{Ec}$ standardised by body mass showed significant differences between all pairs of variables at p 0.001 .

Table 1

Average values of physiological variables during maximal exercise

$\begin{array}{cccc}\mathrm{VO}_{2 m a x} & \mathrm{VO}_{2 m a x} & \text { VEmax } & \text { HRmax } \\ \mathrm{mL}^{-1} \cdot \mathrm{min}^{-1} & \mathrm{~L} \cdot \mathrm{min}^{-1} & \mathrm{~L} \cdot \mathrm{min}^{-1} & \mathrm{sk} \cdot \mathrm{min}^{-1}\end{array}$

$67,4 \pm 7,25 \quad 4,7 \pm 0,81 \quad 139,7 \pm 18,8 \quad 186 \pm 10,7$

$\mathrm{VO}$ max - maximum oxygen uptake, VEmax - maximal lung ventilation, HRmax - maxium heart rate

Table 2

The average energy cost of walking with technical $\left(v_{t}\right)$, threshold $\left(v_{p}\right)$ and racing speeds $\left(v_{s}\right)$

\begin{tabular}{|c|c|c|c|c|c|c|}
\hline \multirow{2}{*}{$\begin{array}{l}\mathrm{V} \\
\mathrm{m} \cdot \mathrm{s}^{-1}\end{array}$} & \multicolumn{6}{|c|}{ Exercise energy cost EE } \\
\hline & $\mathrm{kJ} \cdot \min ^{-1}$ & $\mathrm{kcal} \cdot \mathrm{min}^{-1}$ & $\mathrm{~J} \cdot \mathrm{m}^{-1}$ & $\mathrm{cal} \cdot \mathrm{m}^{-1}$ & $\mathrm{Jm}^{-1} \mathrm{~kg}^{-1}$ & $\mathrm{cal} \cdot \mathrm{m}^{-1} \mathrm{~kg}^{-1}$ \\
\hline $\mathrm{V}_{\mathrm{t}}=3,1 \pm 0,19$ & $51,2 \pm 10,05$ & $12,3 \pm 2,40$ & $267 \pm 52,3$ & $63,8 \pm 12,52$ & $3,86 \pm 0,55$ & $0,92 \pm 0,13$ \\
\hline $\mathrm{v}_{\mathrm{p}}=3,7 \pm 0,13$ & $69,2 \pm 11,04$ & $16,6 \pm 2,64$ & $312 \pm 49,7$ & $74,5 \pm 11,89$ & $4,51 \pm 0,51$ & $1,08 \pm 0,12$ \\
\hline $\mathrm{V}_{\mathrm{s}}=4,0 \pm 0,14$ & $78,3 \pm 13,01$ & $18,8 \pm 3,11$ & $326 \pm 54,2$ & $78,1 \pm 12,97$ & $4,74 \pm 0,62$ & $1,13 \pm 0,15$ \\
\hline
\end{tabular}

Table 3

Average values of $\Delta E_{p}, \Delta E_{k}$ and total external work $\Delta E_{c}$ (the biomechanical cost of walking) with technical $\left(v_{t}\right)$, threshold $\left(v_{p}\right)$, and racing speed $\left(v_{s}\right)$

\begin{tabular}{|c|c|c|c|c|c|c|c|c|c|}
\hline \multirow{2}{*}{$\begin{array}{l}\mathrm{V} \\
\mathrm{m} \cdot \mathrm{s}^{-1}\end{array}$} & \multicolumn{9}{|c|}{ Biomechanical cost of exercise "total energy" } \\
\hline & $\begin{array}{c}\Delta \mathrm{E}_{\mathrm{p}} \\
\mathrm{kJ} \cdot \mathrm{min}^{-1}\end{array}$ & $\begin{array}{c}\Delta \mathrm{Ek} \\
\mathrm{kJ} \cdot \mathrm{min}^{-1}\end{array}$ & $\begin{array}{c}\Delta \mathrm{E}_{\mathrm{c}} \\
\mathrm{kJ} \cdot \mathrm{min}^{-1}\end{array}$ & $\begin{array}{c}\Delta \mathrm{E}_{\mathrm{p} \_s t d} \\
\mathrm{~J} \cdot \mathrm{m}^{-1}\end{array}$ & $\begin{array}{c}\Delta \mathrm{E}_{\mathrm{k} \_s t d} \\
\mathrm{~J} \cdot \mathrm{m}^{-1}\end{array}$ & $\begin{array}{l}\Delta \mathrm{E}_{\mathrm{c} \_s t d} \\
\mathrm{~J} \cdot \mathrm{m}^{-1}\end{array}$ & $\begin{array}{c}\Delta \mathrm{E}_{\mathrm{p} \_s t d} \\
\mathrm{~J} \cdot \mathrm{m}^{-1} \cdot \mathrm{kg}^{-1}\end{array}$ & $\begin{array}{c}\Delta \mathrm{Ek}_{\mathrm{k} \_s t d} \\
\mathrm{~J} \cdot \mathrm{m}^{-1} \cdot \mathrm{kg}^{-1}\end{array}$ & $\begin{array}{c}\Delta \mathrm{E}_{\mathrm{c} \_ \text {std }} \\
\mathrm{J} \cdot \mathrm{m}^{-1} \cdot \mathrm{kg}^{-1}\end{array}$ \\
\hline $\mathrm{V} t_{\mathrm{t}}=3,1 \pm 0,19$ & $\begin{array}{c}4,25 \\
\pm 0,33\end{array}$ & $\begin{array}{c}8,91 \\
\pm 0,83\end{array}$ & $\begin{array}{l}13,16 \\
\pm 1,25\end{array}$ & $\begin{array}{c}22,1 \\
\pm 2,08\end{array}$ & $\begin{array}{c}46,4 \\
\pm 4,12\end{array}$ & $\begin{array}{c}68,6 \\
\pm 5,13\end{array}$ & $\begin{array}{c}0,32 \\
\pm 0,05\end{array}$ & $\begin{array}{c}0,67 \\
\pm 0,11\end{array}$ & $\begin{array}{c}0,99 \\
\pm 0,14\end{array}$ \\
\hline$v_{p}=3,7 \pm 0,13$ & $\begin{array}{c}4,93 \\
\pm 0,42\end{array}$ & $\begin{array}{l}13,05 \\
\pm 1,12\end{array}$ & $\begin{array}{l}18,00 \\
\pm 1,79\end{array}$ & $\begin{array}{c}22,3 \\
\pm 2,06\end{array}$ & $\begin{array}{c}58,8 \\
\pm 4,15\end{array}$ & $\begin{array}{c}81,1 \\
\pm 7,16\end{array}$ & $\begin{array}{c}0,32 \\
\pm 0,06\end{array}$ & $\begin{array}{c}0,85 \\
\pm 0,10\end{array}$ & $\begin{array}{c}1,17 \\
\pm 0,15\end{array}$ \\
\hline $\mathrm{V}_{\mathrm{s}}=4,0 \pm 0,14$ & $\begin{array}{c}5,14 \\
\pm 0,48\end{array}$ & $\begin{array}{l}16,19 \\
\pm 1,37\end{array}$ & $\begin{array}{l}21,33 \\
\pm 1,98\end{array}$ & $\begin{array}{c}21,4 \\
\pm 2,05\end{array}$ & $\begin{array}{c}67,4 \\
\pm 5,20\end{array}$ & $\begin{array}{c}88,9 \\
\pm 8,22\end{array}$ & $\begin{array}{c}0,31 \\
\pm 0,05\end{array}$ & $\begin{array}{c}0,98 \\
\pm 0,13\end{array}$ & $\begin{array}{c}1,29 \\
\pm 0,16\end{array}$ \\
\hline
\end{tabular}




\section{Discussion}

Race walking is an endurance sport discipline, so in addition to the perfect walking technique race walkers must show substantial aerobic endurance expressed by maximum oxygen uptake per minute (Schwartz et al., 2006). Compared with results achieved by athletes in other disciplines, including endurance athletes, average $\mathrm{VO} 2 \mathrm{max}$ of 67.4 mL.kg-1.min-1 achieved in the experiment does not rank among the highest. In the worldclass skiers the variable may even exceed 80 mL.kg-1.min-1 (Bergh, 1982). A similar value has been registered for a four time Olympic gold medallist in race walking during aerobic capacity testing carried out at the Department of Physiology and Biochemistry, of the Academy of Physical Education in Cracow. In this experiment, the participants could effectively compensate for much lower aerobic endurance by being able to walk relatively fast at the anaerobic threshold. An average speed of 3.7 m.s-1 and a comparatively high anaerobic threshold allowed them to perform at a high level during domestic as well as international competitions.

The racing speed of $4.0 \mathrm{~m} . \mathrm{s}-1$ the participants achieved is 2.3 times as high as the speed of individually selected fast walk of male nonathletes aged 20-59 years, which is $1.8 \mathrm{~m} . \mathrm{s}-1$ on average (Waters et al., 1988). Oxygen uptake in a functional steady state at this walking speed amounted to an average of 18.4 mL.kg-1.min-1 and the heart rate was 124 beats.min-1 (Waters et al., 1983). In participants who walked twice as fast, the minute oxygen uptake was ca. $67.4 \pm 7.26$ mL.kg-1.min-1 and the pulse rate was $186 \pm 18.8$ beats per .min-1. Particularly noteworthy is that the participants had lower physiological (aerobic) cost of walking per meter of distance covered (0.17 mL.kg-1.m-1) compared with non-athlete males walking with an almost twice lower speed (0.19 mL.kg-1.m-1).

Total external work of muscles necessary to accelerate and lift the centre of body mass was significantly different from the energy cost EE and its values were distinctly lower. For technical and threshold speeds it accounted for ca $26 \%$ and for racing speed for $27 \%$ of the energy cost obtained. According to Duff-Raffaele et al. (1996), an analogous proportion of external muscle work in the metabolic cost of a physiological gait at normal speed exceeds $50 \%$. Total external work is exclusive of cost components such as the storage and release of elastic energy (Ishikawa et al., 2005), transfer of energy between the body segments (Bastien et al., 2003), kinetic energy of the rotation of body segments about the biomechanical axis of the joint, or the cost of cocontractions of antagonist muscles and of postural stabilisation. Neptune et al. (2008) state, however, that the total external work of muscles done to lift and accelerate $\mathrm{CoM}$ is significantly correlated with metabolic cost.

Total external work of muscles necessary to ensure correct control of CoM displacements shows the nature of the technical gait pattern for particular speeds. Chwała (2013) argues that the amount of energy race walkers can recoup through the inverse pendulum mechanism is small, below $20 \%$. Therefore, minimization of total external work is a proof of an optimised gait technique and indirectly helps reduce energy cost. Significant differences in total external work of muscles in mean values, absolute and standardised by one meter of distance covered and one kilogram of body mass have been found in the experiment ( $p$ 0,001) regardless of walking speed. This reflects that the biomechanical cost increases significantly along with walking speed. Walking speed increasing from technical to racing involved clearly bigger changes in the kinetic energy of the centre of mass related to its acceleration and deceleration. At the same time, changes in potential energy related to the vertical oscillations of the centre of mass remained at a similar level (Table 3).

There have been significant differences between the mean values of physiological cost of walking for all speeds, expressed in kJ.m-1 (p 0,001). In addition, we noted significantly different average physiological cost standardized to distance covered (J.m-1) and body mass (J.m-1kg-1), between the technical and threshold speeds as well as the technical and racing speeds (p 0,005). Significant differences were registered between all considered physiological and biomechanical variables characterizing the energy cost of race walking (p 0,001). Mean absolute values of EE and $\Delta \mathrm{Ec}$ (expressed in $\mathrm{kJ} \bullet \mathrm{min}-1$ ) were significantly different for the speed threshold (p 0,05) and home (p 0,005).

Regarding energy cost, the threshold speed and racing speed were not significantly different from 
each other. On the other hand, a speed increase from threshold to racing had a similar proportion of the technical component in total cost.

Comparing the mean standardized values of the physiological and biomechanical cost we noted significant differences between all the analyzed variables ( $p$ 0,001). Mean absolute values of EE and $\Delta \mathrm{Ec}$ (expressed in kJ.min-1) were significantly different for the threshold $(p 0,05)$ and racing (p 0,005) speed.

A noteworthy finding is that both energy cost and biomechanical cost were much lower in athletes walking at the technical speed, which allows for an optimal gait pattern to be implemented. However, to perform well in race walking events, the speeds must be significantly higher, slightly above the threshold speed, but then the gait becomes more "costly". Therefore, individual movement technique should be optimised towards minimal vertical oscillations of the centre of mass combined with a smooth passage from the heel-strike to toe-off phase involving only small changes in kinetic energy. The results also clearly indicate that the other components of mechanical energy mentioned above account for a considerable portion of total energy cost. This mean that all factors likely to influence total energy cost must be considered for a movement technique to be correct.
The presented results are important for the practice of sport training, as they enable the optimisation of individual walking technique towards minimum energy cost.

\section{Conclusions}

1. High levels of maximum oxygen uptake and relatively high threshold and racing speeds in the studied athletes showed their high aerobic capacity characterising the ability to perform endurance exercise.

2. The analysis indicated significant differences between the average values of energy costs and total external work calculated for the technical speed, threshold and racing speeds.

3. Total external work of muscles computed in the experiment accounted for around one fourth of total energy cost in race walking regardless of the type of speed.

4. The most important for the total external work increase were changes in the resultant kinetic energy of the centre of mass.

5. In the classification of physical exercise by energy cost race walking with a technical speed is placed in the upper zone of ",very heavy" exercise, whereas walking with threshold and racing speeds is recognised as "extremely heavy".

6. The results of the present study can be used to design training activities aiming at the optimisation of energy cost in race walking.

\section{Acknowledgements}

This scientific research was financed on the research project NCN NN404202837

\section{References}

Bastien GJ, Heglund NC, Schepens B. The double contact phase in walking children. J. Exp. Biol, 2003; 206: 2967-2978

Bergh U. Physiology of cross-country ski racing. Champaign. Human Kin. Publ, 1982

Cavagna GA, Willems PA, Legramandi MA, Heglund NC. Pendular energy transduction within the step in human walking. J. Exp. Biol, 2002; 205(21): 3413-3422

Chwała W. Effect of speed on changes positions of the body's center of gravity and efficiency energy recovery mechanism in physiological gait and racewalking. Wyd. Monograf. Akademii Wychowania Fizycznego w Krakowie 12, 206-254; 2013

Duff-Raffaele M, Kerrigan DC, Corcoran PJ, Saini M. The proportional work of lifting the center of mass during walking. Am. J. Phys. Med. Rehabil, 1996; 75: 375-9

Eames MHA, Cosgrove A, Baker R. Comparing methods of estimating the total body centre of mass in threedimensions in normal and pathological gaits. Hum. Mov. Sci, 1999; 18(5): 637-646

Ishikawa M, Komi P, Grey M, Lepola V, Bruggemann G. Muscle-tendon interaction and elastic energy usage in human walking. J. Appl. Physiol, 2005; 99(2): 603-608 
Klimek AT, Chwała W. The evaluation of energy cost of effort and changes of centre of mass (COM) during race walking at starting speed after improving the length of lower extremities. Acta Bioeng. Biomech, 2007; 9(2): 55-60

Minetti AE, Ardigo LP, Saibene F. The transition between walking and running in humans: metabolic and mechanical aspects at different gradients. Acta Physiol. Scand, 1994; 150: 315-323

Neptune RR, Sasaki K, Kautz SA. The effect of walking speed on muscle function and mechanical energetics. Gait Post, 2008; 28: 135-143

Ortega JD, Farley CT. Minimizing center of mass vertical movement increases metabolic cost in walking. J. Appl. Physiol. 2005; 99: 2099-2107

Reinhard U, Müller PH, Schmulling RM. Determination of anaerobic threshold by the ventilation equivalent in normal individuals. Respiration, 1979; 38: 36

Schepens B, Bastien GJ, Heglund NC, Willems PA. Mechanical work and muscular efficiency in walking children. J. Exp. Biol, 2004; 207: 587-596

Schwartz MH, Koop SE, Bourke JL, Baker R. A nondimensional normalization scheme for oxygen utilization data. Gait Post, 2006; 24(1): 14-22

Umberger BR, Martin PE. Mechanical power and efficiency of level walking with different stride rates. J. Exp. Biol., 2007; 210: 3255-3265

Van de Walle P, Desloovere K, Truijen S, Gosselink R, Aerts P, Hallemans A. Agerelated changes in mechanical and metabolic energy during typical gait. Gait Post, 2010; 31(4): 495-501

Waters RL, Hislop HJ, Perry J, Thomas L, Campbell J. Comparative cost of walking in young and old adults. J. Orthop. Res, 1983; 1(1): 73-76

Waters RL, Lunsford BR, Perry J, Byrd R. Energy - speed relationship of walking: standard tables. J. Orthop. Res, 1988; 6(2): 215-222

\section{Corresponding author:}

Wiesław Chwała,

Institute of Biomedical Sciences, Department of Biomechanics of University School of Physical Education in Cracow, Poland.

Al. Jana Pawla II 78,

31-571 Cracow, Poland

Phone/fax +48126831133

E-mail: wieslaw.chwala@awf.krakow.pl; wachwala@cyf-kr.edu.pl 